\title{
Microscopic Calculation of Pre-Compound Excitation Energies for Heavy-Ion Collisions
}

\author{
A.S. Umar, ${ }^{1}$ V.E. Oberacker, ${ }^{1}$ J.A. Maruhn, ${ }^{2}$ and P.-G. Reinhard ${ }^{3}$ \\ ${ }^{1}$ Department of Physics and Astronomy, Vanderbilt University, Nashville, Tennessee 37235, USA \\ ${ }^{2}$ Institut für Theoretische Physik, Goethe-Universität, 60438 Frankfurt am Main, Germany \\ ${ }^{3}$ Institut für Theoretische Physik, Universität Erlangen, D-91054 Erlangen, Germany
}

(Dated: May 21, 2018)

\begin{abstract}
We introduce a microscopic approach for calculating the excitation energies of systems formed during heavy-ion collisions. The method is based on time-dependent Hartree-Fock (TDHF) theory and allows the study of the excitation energy as a function of time or ion-ion separation distance. We discuss how this excitation energy is related to the estimate of the excitation energy using the reaction $Q$-value, as well as its implications for dinuclear pre-compound systems formed during heavy-ion collisions.
\end{abstract}

PACS numbers: 21.60.-n,21.60.Jz

During a heavy-ion collision the initial kinetic energy of the two nuclei is gradually converted into internal excitation. This excitation may result in exciting various types of collective modes or intrinsic excitations that lead to the heating of the combined nuclear system. The excitation of projectile-like and target-like fragments in deep-inelastic heavy-ion collisions is a well known manifestation of this phenomenon, which has been studied theoretically and experimentally [1]. On the other hand, for a dinuclear pre-compound system formed during a heavy-ion collision, the mode of decay may critically depend on the excitation energy of the system. Examples include collisions that may be candidates for the formation of superheavy elements in hot or cold fusion reactions [2, 3]. Excitation energy is also an important ingredient for the stochastic mean-field approach to nuclear dynamics, which deals with fluctuations of collective motion in addition to the average evolution [4, 5]. While exclusive measurements of excitation energy may be possible for equilibrated systems (e.g. compound nuclei formed in complete fusion or fragments produced in deep-inelastic collisions), the intermediate states formed during a collision have a short life time and are not expected to be fully equilibrated thus making the measurement as well as the interpretation very difficult [6]. For all these reasons it is desirable to develop a dynamical approach for calculating the excitation energy of the system as it evolves in time.

It is generally acknowledged that the TDHF theory provides a useful foundation for a fully microscopic manybody theory of low-energy heavy-ion reactions [7, 8]. While the long-time evolution in TDHF theory may not be very reliable, recent three-dimensional TDHF calculations with no symmetry assumptions and using modern Skyrme forces have shown to accurately reproduce phenomena determined by the initial stages of the heavyion dynamics [9, 10, 11]. Recently we have developed the density-constrained TDHF (DC-TDHF) method [12], which is based on the generalization of the density constraint method developed earlier [13]. We have shown that using the DC-TDHF method ion-ion potential barriers can be accurately produced [14, 15, 16] as these calculations also depend on early stages of the ion-ion dynamics. In addition, one-body energy dissipation extracted from TDHF for low-energy fusion reactions was found to be in agreement with the friction coefficients based on the linear response theory as well as those in models where the dissipation was specifically adjusted to describe experiments [17]. All of these new results suggest that TDHF dynamics provide a good description of the early stages of heavy-ion collisions.

In this manuscript we outline a microscopic approach for calculating excitation energies of composite or dinuclear systems formed during heavy-ion collisions. The goal of the approach is to divide the TDHF motion into a collective and intrinsic part. The major assumption in achieving this goal is to assume that the collective part is primarily determined by the density $\rho(\mathbf{r}, t)$ and the current $\mathbf{j}(\mathbf{r}, t)$. Consequently, the excitation energy can be formally written as

$$
E^{*}(t)=E_{T D H F}-E_{\text {coll }}(\rho(t), \mathbf{j}(t)),
$$

where $E_{T D H F}$ is the total energy of the dynamical system, which is a conserved quantity, and $E_{\text {coll }}$ represents the collective energy of the system. In the next step we break up the collective energy into two parts

$$
E_{\text {coll }}(t)=E_{\text {kin }}(\rho(t), \mathbf{j}(t))+E_{D C}(\rho(t)),
$$

where $E_{k i n}$ represents the kinetic part and is given by

$$
E_{k i n}(\rho(t), \mathbf{j}(t))=\frac{m}{2} \int \mathrm{d}^{3} r \mathbf{j}^{2}(t) / \rho(t),
$$

which is asymptotically equivalent to the kinetic energy of the relative motion, $\frac{1}{2} \mu \dot{R}^{2}$, where $\mu$ is the reduced mass and $R(t)$ is the ion-ion separation distance. The energy $E_{D C}$ is the lowest-energy state of all possible TDHF states with the same density and is required to have zero excitation energy. This state is found by using the density-constraint method [13, 18], which minimizes 
the energy while holding the instantaneous TDHF density constant. We have previously shown [12] that $E_{D C}$ equals the ion-ion potential, $V(R)$, shifted by the binding energies of the participating nuclei, which allows us to write

$$
E_{\text {coll }}(t)=E_{\text {kin }}(\rho(t), \mathbf{j}(t))+V(R(t))+E_{A_{1}}+E_{A_{2}},
$$

where $E_{A_{1}}$ and $E_{A_{2}}$ denote the Hartree-Fock energies calculated for the two nuclei using the same effective interaction. The dynamics of the ion-ion separation $R(t)$ can be extracted from an unrestricted TDHF run. Using $E^{*}(t)$ and $R(t)$, we can deduce the excitation energy as a function of the distance parameter, $E^{*}(R)$.

The computation of the excitation energy as outlined above is numerically very intensive, primarily due to the density-constraint calculation. Calculations were done in 3-D geometry and using the full Skyrme force (SLy4) 19] without the center-of-mass correction as described in Ref. [20]. The numerical accuracy of the static binding energies and the deviation from the point Coulomb energy in the initial state of the collision dynamics is on the order of $50-200 \mathrm{keV}$. We have performed density constraint calculations at every $20 \mathrm{fm} / \mathrm{c}$. For the calculation of the ion-ion separation distance $R$ we use the hybrid method, which relates the coordinate to the quadrupole moment for small $R$ values, as described in Ref. [16]. The accuracy of the density constraint calculations is commensurate with the accuracy of the static calculations.

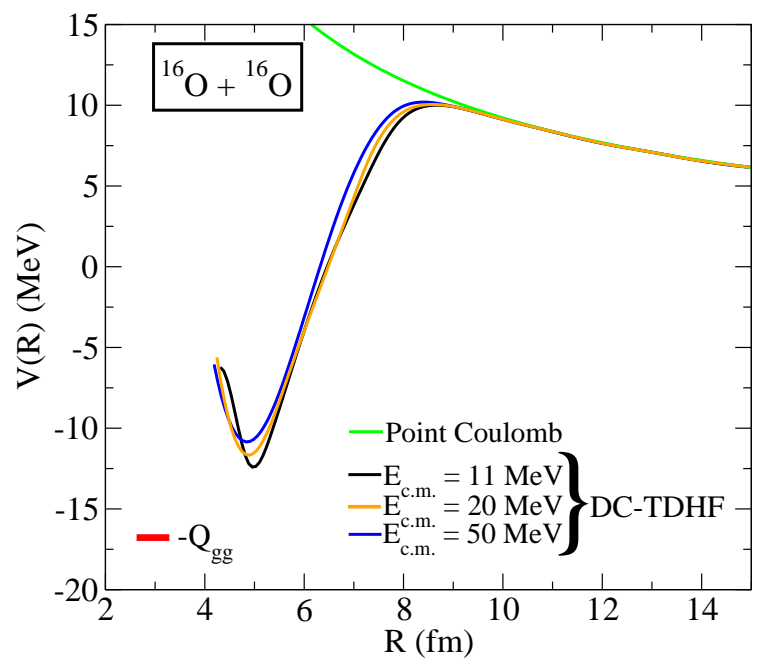

FIG. 1: Internuclear potential $V(R)$ for the head-on collision of the ${ }^{16} \mathrm{O}+{ }^{16} \mathrm{O}$ system for various $E_{\text {c.m. values. The relative }}$ ground-state binding energy of the ${ }^{32} \mathrm{~S}$ nucleus is represented by the $-Q_{g g}$ value.

In order to develop a better insight into the excitation energy given by Eq. (11) we have first studied two spherical systems, ${ }^{16} \mathrm{O}+{ }^{16} \mathrm{O}$ and ${ }^{40} \mathrm{Ca}+{ }^{40} \mathrm{Ca}$. In literature one commonly defines the excitation energy for a particular reaction as

$$
E^{*}=E_{c . m .}+Q_{g g}
$$

where $Q_{g g}$ is the mass difference between the two initial nuclei and the combined system in its ground state. While this expression is correct relative to the ground state of the composite system, it does not accurately describe the excitation energy relative to other intermediate transition states formed during the collision. Our choice of the reactions mentioned above was motivated by the fact that the former system has a positive $Q_{g g}$ value $(16.6 \mathrm{MeV})$, whereas the latter system has a negative one $(-14.2 \mathrm{MeV})$.

In Fig. 1 we show the ion-ion interaction potential $V(R)$ for the head-on (zero impact parameter) collision of the ${ }^{16} \mathrm{O}+{ }^{16} \mathrm{O}$ system at various center-of-mass energies. These results are essentially the same as those published in Ref. [12] except for the energy dependence of $V(R)$. This dependence arises from the time available for the system to undergo rearrangements and partial equilibration, which approaches the frozen-density limit at high energies [17]. On the same figure we have also shown the

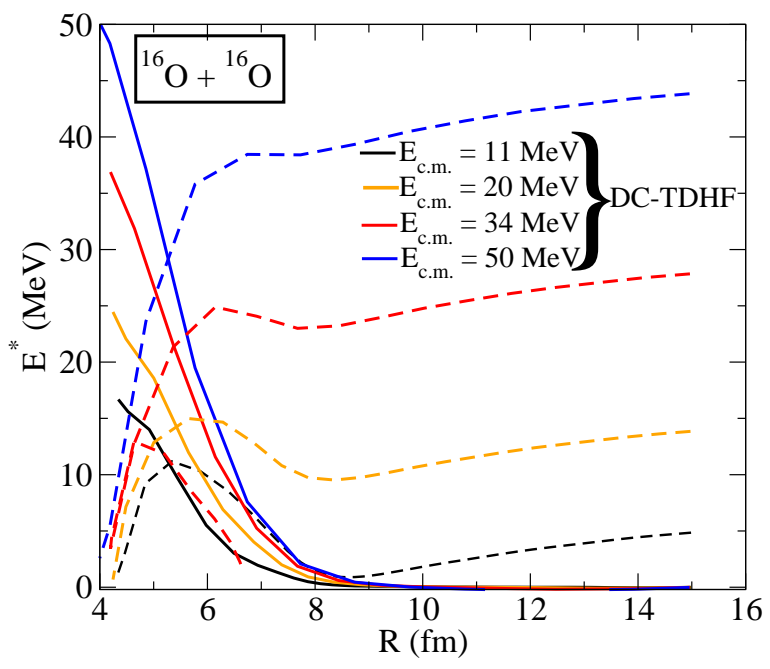

FIG. 2: Excitation energy for the head-on collision of the ${ }^{16} \mathrm{O}+{ }^{16} \mathrm{O}$ system for various $E_{\text {c.m. values (solid curves) as }}$ a function of the ion-ion distance $R$. Also, shown are the corresponding collective kinetic energy $E_{k i n}$ values (dashed curves).

relative location of the ${ }^{32} \mathrm{~S}$ ground-state binding energy as represented by the $-Q_{g g}$ value. In Fig. 2 the corresponding excitation energies are shown as a function of $R$ calculated from Eq. (11). The fact that the excitation energy should be asymptotically zero is a good test of numerical accuracy, which is reproduced quite well as can been seen from the figure. The final value of $E^{*}$ and smallest $R$ value are chosen to be the ones corresponding to the smallest relative velocity or smallest collective kinetic energy in the vicinity of the potential minimum, which alternately can be referred to as the capture point. Naturally, some of these quantities can only be pinned down within the limits of density-constraint frequency. For sake of completeness in Fig. 3 we also show the longtime behavior of the potential and the excitation energy 


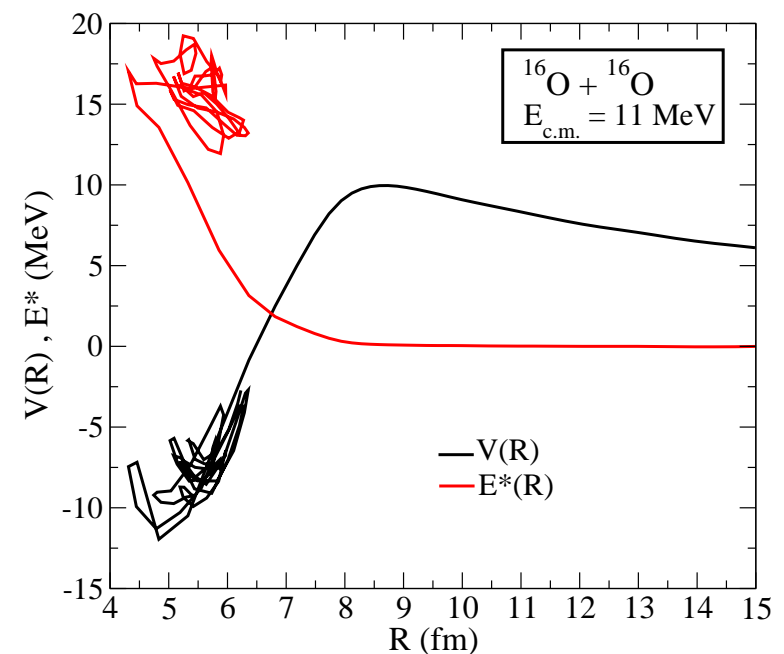

FIG. 3: Long-time evolution of the excitation energy, $E^{*}$, and the ion-ion potential, $V(R)$, for the head-on collision of the ${ }^{16} \mathrm{O}+{ }^{16} \mathrm{O}$ system at $E_{\text {c.m. }}=11 \mathrm{MeV}$ as a function of the ion-ion distance $R$.

for the ${ }^{16} \mathrm{O}+{ }^{16} \mathrm{O}$ system at $E_{\text {c.m. }}=11 \mathrm{MeV}$. The figure demonstrates very nicely that the majority of the entrance energy is absorbed into intrinsic motion such that the compound stage is stuck in the vicinity of a certain $R$ value with rather small oscillations. In the entrance phase of the collision, the excitation energy $E^{*}$ increases monotonically with decreasing $R$. After the point of closest approach has been reached, the system is stuck close to that distance and the dynamical evolution turns abruptly to irregular oscillations in $E^{*}$ as seen in Fig. 3. which the typical energy fluctuations of an excited ensemble. These fluctuations are here rather large due to

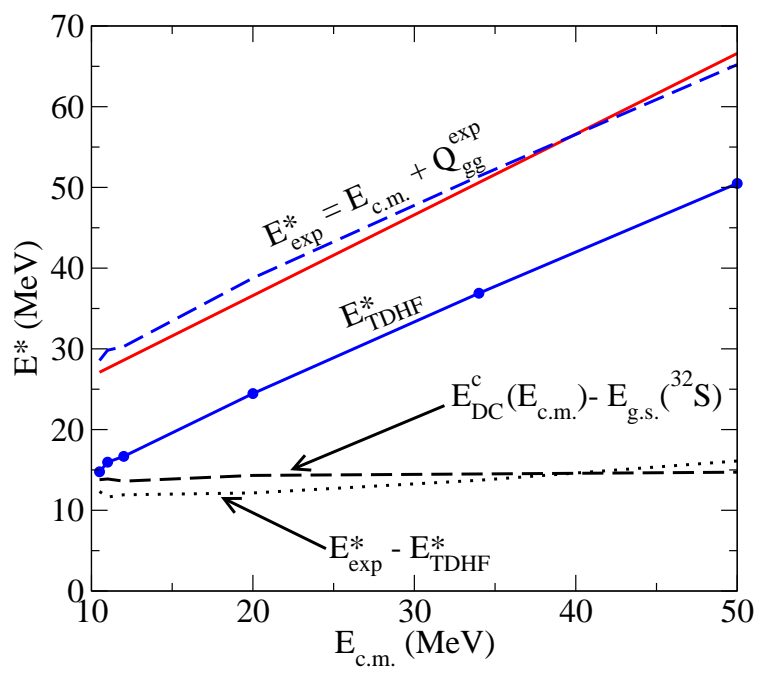

FIG. 4: Excitation energy for the head-on collision of the ${ }^{16} \mathrm{O}+{ }^{16} \mathrm{O}$ system for various $E_{\text {c.m. }}$. values at the point of capture (solid blue line) and the excitation energy calculated from Eq. (5) (solid red curve). Other curves are described in the manuscript. the small particle number. The statistical estimate for the energy width is $\Delta E \approx \sqrt{16 \varepsilon_{\mathrm{F}} E^{*} /\left(N \pi^{2}\right)} \approx 6 \mathrm{MeV}$, which fits nicely to the observed fluctuations.

Next we wanted to demonstrate the conjecture that the value of the excitation energy measured at the capture point in TDHF is the excitation relative to the composite or dinuclear system formed during the collision. In Fig. 4 we plot the excitation energy as a function of the center-of-mass energy for both the analytic expression of Eq. (5) (solid red line) and the TDHF results at the capture point as discussed above (solid blue line). As expected the TDHF result is below the one generated from Eq. (5). The difference between the two curves is shown by the dotted line. We have also calculated the energy difference of the composite system relative to the ground state, which is simply the $E_{D C}$ at the point of capture minus the ground state energy of the ${ }^{32} \mathrm{~S}$ system obtained by an unconstrained Hartree-Fock calculation. This is shown by the black dashed curve. If we shift

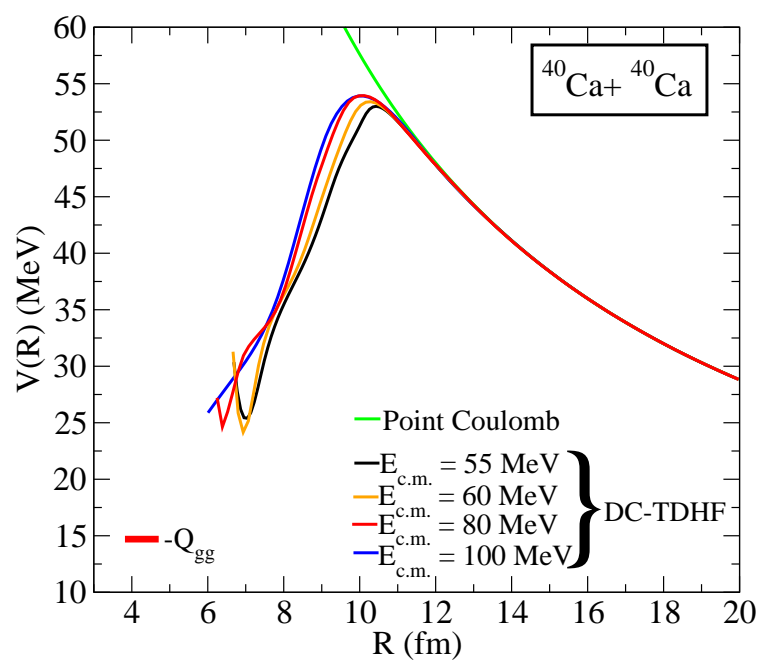

FIG. 5: Internuclear potential for the head-on collision of the ${ }^{40} \mathrm{Ca}+{ }^{40} \mathrm{Ca}$ system for various $E_{\text {c.m. values. The relative }}$ ground state binding energy of the ${ }^{80} \mathrm{Zr}$ nucleus is represented by the $-Q_{g g}$ value.

the TDHF result by these differences we obtain the blue dashed curve, which is in agreement with the result obtained from Eq. (5). We should emphasize at this point that for many reactions the excitation energy at the capture point is of great interest as opposed to the ground state to ground state value, since after the capture point many different reaction possibilities exist.

We have repeated the above study for the ${ }^{40} \mathrm{Ca}+{ }^{40} \mathrm{Ca}$ system, for which the $Q_{g g}$ value is $-14.2 \mathrm{MeV}$. In Fig. 15 we show the ion-ion potentials calculated using the DCTDHF method at a set of center-of-mass energies. The energy dependence is analogous to the ${ }^{16} \mathrm{O}+{ }^{16} \mathrm{O}$ case. Fig. [6] shows the corresponding excitation energies calculated via TDHF using Eq. (11). Again, the excitation energies gradually rise as the nuclei enter the interaction regime while the collective kinetic energies show a 
rise when the nuclei first experience the nuclear attraction but eventually fall due to slowdown of the composite system. Finally, we again plot the center-of-mass energy

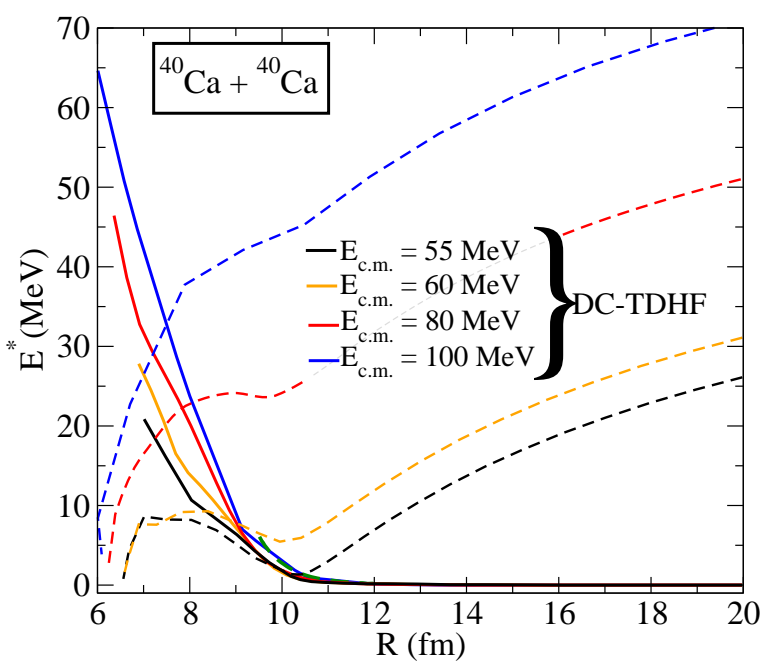

FIG. 6: Excitation energy for the head-on collision of the ${ }^{40} \mathrm{Ca}+{ }^{40} \mathrm{Ca}$ system for various $E_{\text {c.m. values (solid curves) as }}$ a function of the ion-ion distance $R$. Also, shown are the corresponding collective kinetic energy $E_{k i n}$ values (dashed curves).

dependence of the excitation energy at the point of capture in Fig. 7 (blue solid curve) together with the one obtained from Eq. (5), except this time using the negative $Q_{g g}$ value (solid red line). Again the two curves run

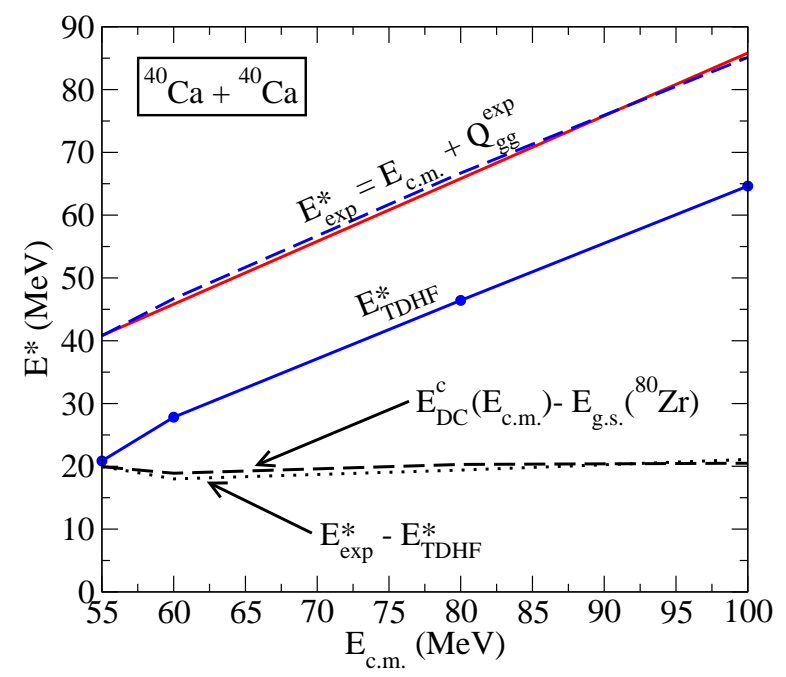

FIG. 7: Excitation energy for the head-on collision of the ${ }^{40} \mathrm{Ca}+{ }^{40} \mathrm{Ca}$ system for various $E_{\text {c.m. }}$. values at the point of capture (solid blue line) and the excitation energy calculated from Eq. (5) (solid red curve). Other curves are described in the manuscript.

parallel to each other and their difference is shown by the dotted line. The long dashed line shows the difference in energy between the density constrained energy $E_{D C}$ at the point of capture minus the ground state energy of the ${ }^{80} \mathrm{Zr}$ nucleus. The two curves are almost exactly the same and shifting the TDHF excitation curve by this energy difference produces the blue dashed curve, which is in excellent agreement with the one obtained using Eq. (5). Thus, we can safely say that the microscopically calculated excitation energy represents the excitation energy at the point of capture.

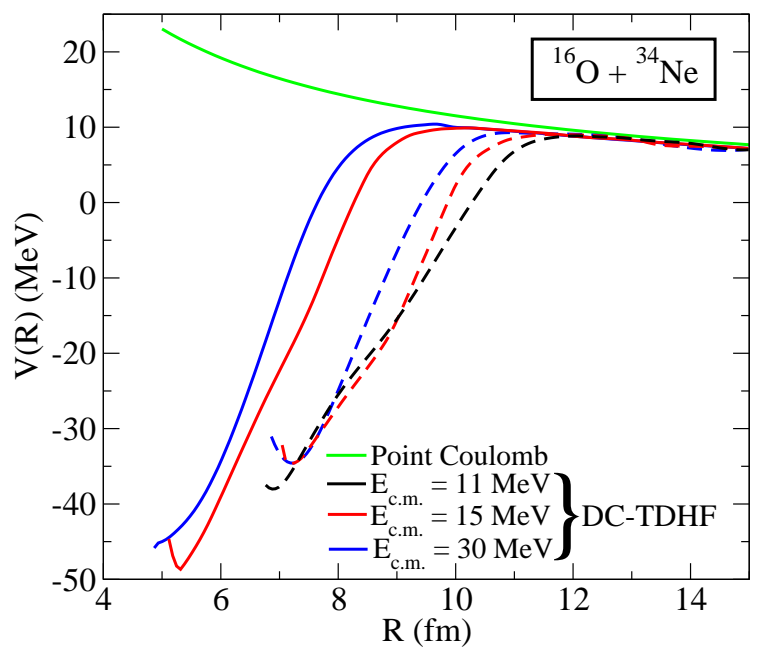

FIG. 8: Internuclear potential for the head-on collision of the ${ }^{16} \mathrm{O}+{ }^{34} \mathrm{Ne}$ system for various $E_{\text {c.m. }}$. values and two alignments of the ${ }^{34} \mathrm{Ne}$ nucleus. The solid lines denote the potential for vertical alignment whereas the dashed curves are for the horizontal alignment of the ${ }^{34} \mathrm{Ne}$ nucleus with respect to the collision axis.

It is also possible to obtain an approximate temperature by relating the excitation energy to the temperature using the Fermi gas model, $E^{*}=a T^{2}$, where $a \approx$ $A / 8 \mathrm{MeV}^{-1}$ is the level density parameter. For the above reactions and the $E_{\text {c.m. }}$ values used, this translates into a temperature range of $2.0-3.5 \mathrm{MeV}$ for the ${ }^{16} \mathrm{O}+{ }^{16} \mathrm{O}$ system and a temperature range of $1.4-2.5 \mathrm{MeV}$ for the ${ }^{40} \mathrm{Ca}+{ }^{40} \mathrm{Ca}$ system. The reliability of the above approximation should be higher for heavier systems.

In order to examine how deformation influences the excitation energy during a heavy-ion collision we have chosen to investigate the ${ }^{16} \mathrm{O}+{ }^{34} \mathrm{Ne}$ system. In Hartree-Fock calculations the neutron-rich ${ }^{34} \mathrm{Ne}$ nucleus has a large axially symmetric deformation. In the past we have examined the effects of deformation on the ion-ion potentials due to the different initial alignments of the deformed nucleus [12, 21]. We have performed TDHF collisions of ${ }^{16} \mathrm{O}+{ }^{34} \mathrm{Ne}$ at various center-of-mass energies and for the two extreme alignments of the ${ }^{34} \mathrm{Ne}$ nucleus, one in which the elongation axis is along the collision axis and the other for which it is perpendicular. In Fig. 8 we show the ion-ion potentials obtained for this system using the DC-TDHF method. We observe that at $E_{\text {c.m. }}=11 \mathrm{MeV}$ the vertical alignment case does not fuse, whereas the horizontal alignment does. Furthermore, the centroids of the potentials are different for the two alignments since 
the nuclei start to come into contact earlier/later for horizontal/vertical alignments. Otherwise the energy dependence of the potentials is commensurate with the spherical systems. The fact that different orientations of the deformed nucleus lead to a difference in the time of contact between the target and the projectile is expected to manifest itself in the evolution of the excitation energy as well. Indeed, in Fig. 9 we see that the excitation for

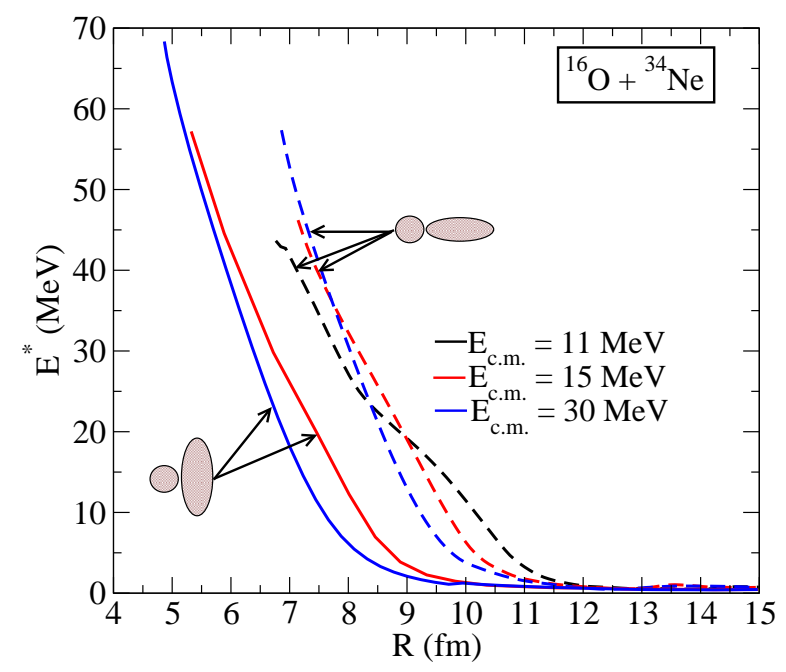

FIG. 9: Excitation energy for the head-on collision of the ${ }^{16} \mathrm{O}+{ }^{34} \mathrm{Ne}$ system for various $E_{\text {c.m. }}$. values as a function of the ion-ion distance $R$. The solid lines denote the excitation energy for vertical alignment whereas the dashed curves are for the horizontal alignment of the ${ }^{34} \mathrm{Ne}$ nucleus with respect to the collision axis. the horizontal alignment of the ${ }^{34} \mathrm{Ne}$ nucleus starts earlier but somehow does not reach as large a value as the vertical alignment case. This is a very interesting observation since it would indicate that due to the differences in the excitation energy at the point of capture different alignments will likely have different probabilities for various exit channels. For example, this could be a very important consideration for superheavy formations.

In summary, we have outlined a microscopic approach for calculating excitation energies of composite or dinuclear systems formed during heavy-ion collisions. The goal of the approach is to provide estimates for excitation energies at the initial point of capture, after which a multitude of exit-channel possibilities may exist and will be strongly influenced by the amount of excitation. The premise of our approach depends on the reliability of TDHF theory for describing the early stages of heavyion reactions. As discussed in the manuscript, there is mounting evidence that TDHF does provide a reliable description of heavy-ion dynamics and dissipation in this stage whereas the long-time evolution may be questionable. In order to elucidate the above arguments we have performed a number of calculations involving both spherical and deformed nuclei. It is our long-term goal to extend these calculations to heavier systems and in particular to superheavy nuclei.

This work has been supported by the U.S. Department of Energy under grant No. DE-FG02-96ER40963 with Vanderbilt University, and by the German BMBF under contracts Nos. 06FY159D and 06ER142D.
[1] J. Töke and W. U. Schröder, Annu. Rev. Nucl. Part. Sci. 42, 401 (1992).

[2] S. Hofmann et al., Eur. Phys. J. A 14, 147 (2002).

[3] Yuri Oganessian, J. Phys. G: Nucl. Part. Phys. 34, R165 (2007).

[4] S. Ayik, Phys. Lett. B 658, 174 (2008).

[5] S. Ayik, K. Washiyama and D. Lacroix, Phys. Rev. C 79, 054606 (2009).

[6] D.Brink, Nucl. Phys. A 519, 3c-16c (1990).

[7] J. W. Negele, Rev. Mod. Phys. 54, 913 (1982).

[8] K. T. R. Davies, K. R. S. Devi, S. E. Koonin, and M. R. Strayer, in Treatise on heavy ion Science, edited by D. A. Bromley, Plenum, New York, 1985), Vol.3, page 3.

[9] Lu Guo, J.A. Maruhn, P.-G. Reinhard, and Y. Hashimoto, Phys. Rev. C 77, 041301(R) (2008).

[10] A.S. Umar and V. E. Oberacker, J. Phys. G: Nucl. Part. Phys. 36, 025101 (2009).

[11] A.S. Umar, V. E. Oberacker, and J.A. Maruhn, Eur. Phys. J. A 37, 245 (2008).

[12] A. S. Umar and V. E. Oberacker, Phys. Rev. C 74, 021601(R) (2006).
[13] R. Y. Cusson, P. -G. Reinhard, M. R. Strayer, J. A. Maruhn, and W. Greiner, Z. Phys. A 320, 475 (1985).

[14] A. S. Umar and V. E. Oberacker, Phys. Rev. C 74, 061601(R) (2006).

[15] A. S. Umar and V. E. Oberacker, Phys. Rev. C 77, 064605 (2008).

[16] A.S. Umar and V. E. Oberacker, Eur. Phys. J. A 39, 243 (2009).

[17] Kouhei Washiyama, Denis Lacroix, and Sakir Ayik, Phys. Rev. C 79, 024609 (2009).

[18] A. S. Umar, M. R. Strayer, R. Y. Cusson, P. -G. Reinhard, and D. A. Bromley, Phys. Rev. C 32, 172 (1985).

[19] E. Chabanat, P. Bonche, P. Haensel, J. Meyer and R. Schaeffer, Nucl. Phys. A635, 231 (1998); Nucl. Phys. A643, 441(E) (1998).

[20] A. S. Umar and V. E. Oberacker, Phys. Rev. C 73, 054607 (2006).

[21] A. S. Umar and V. E. Oberacker, Phys. Rev. C 74, 024606 (2006). 